\title{
Large-scale lipidomics identifies associations between plasma sphingolipids and T2DM incidence
}

\author{
Wee Siong Chew, ${ }^{1}$ Federico Torta, ${ }^{2,3}$ Shanshan Ji, ${ }^{3}$ Hyungwon Choi, ${ }^{4,5,6}$ Husna Begum, ${ }^{2,3}$ \\ Xueling Sim, ${ }^{4}$ Chin Meng Khoo, ${ }^{5}$ Eric Yin Hao Khoo, ${ }^{5}$ Wei-Yi Ong, ${ }^{7}$ Rob M. Van Dam, ${ }^{4,5}$ \\ Markus R. Wenk, ${ }^{2,3}$ E. Shyong Tai, ${ }^{4,5,8}$ and Deron R. Herr ${ }^{1,9}$ \\ 'Department of Pharmacology and 'Department of Biochemistry, Yong Loo Lin School of Medicine, National University \\ of Singapore, Singapore. ${ }^{3}$ Singapore Lipidomics Incubator, Life Sciences Institute, National University of Singapore, \\ Singapore. ${ }^{4}$ Saw Swee Hock School of Public Health, National University of Singapore, Singapore. ${ }^{5}$ Department of \\ Medicine, Yong Loo Lin School of Medicine, National University of Singapore and National Health System, Singapore. \\ ${ }^{6}$ Institute of Molecular and Cell Biology, Agency for Science, Technology, and Research, Singapore. ${ }^{7}$ Department of \\ Anatomy, Yong Loo Lin School of Medicine, National University of Singapore, Singapore. ${ }^{8}$ Duke-NUS Graduate Medical \\ School, Singapore. ${ }^{9}$ Department of Biology, San Diego State University, San Diego, California, USA.
}

BACKGROUND. Sphingolipids (SPs) are ubiquitous, structurally diverse molecules that include ceramides, sphingomyelins (SMs), and sphingosines. They are involved in various pathologies, including obesity and type 2 diabetes mellitus (T2DM). Therefore, it is likely that perturbations in plasma concentrations of SPs are associated with disease. Identifying these associations may reveal useful biomarkers or provide insight into disease processes.

METHODS. We performed a lipidomics evaluation of molecularly distinct SPs in the plasma of 2302 ethnically Chinese Singaporeans using electrospray ionization mass spectrometry coupled with liquid chromatography. SP profiles were compared to clinical and biochemical characteristics, and subjects were evaluated with follow-up visits for 11 years.

RESULTS. We found that ceramides correlated positively but hexosylceramides correlated negatively with BMI and homeostatic model assessment of insulin resistance (HOMA-IR). Furthermore, SPs with a d16:1 sphingoid backbone correlated more positively with BMI and HOMAIR, while d18:2 SPs correlated less positively, relative to canonical d18:1 SPs. We also found that higher concentrations of 2 distinct SMs were associated with a higher risk of T2DM (HR 1.45 with 95\% Cl 1.18-1.78 for SM d16:1/18:0 and HR 1.40 with 95\% Cl 1.17-1.68 for SM d18:1/18:0).

CONCLUSIONS. We identified significant associations between SPs and obesity/T2DM characteristics, specifically, those of hexosylceramides, d16:1 SPs, and d18:2 SPs. This suggests that the balance of SP metabolism, rather than ceramide accumulation, is associated with the pathology of obesity. We further identified 2 specific SPs that may represent prognostic biomarkers for T2DM.

FUNDING. National University Health System (NUHSRO/2014/085/AF-Partner/01) and the National Research Foundation Investigatorship grant (NRF-NRFI2015-05).

Authorship note: WSC and FT contributed equally to this work.

Conflict of interest: The authors have declared that no conflict of interest exists.

Copyright: (c) 2019 American Society for Clinical Investigation

Submitted: December 19, 2018

Accepted: May 28, 2019

Published: June 4, 2019.

Reference information: JCI Insight. 2019;4(13):e126925. https://doi. org/10.1172/jci.insight.126925.

\section{Introduction}

Research over the past few decades has vastly improved our understanding of the pathogenesis of type 2 diabetes mellitus (T2DM). The advent of increasingly sophisticated metabolomics techniques using mass spectrometry has contributed to this improvement considerably. However, our understanding of the molecular events leading to the onset of T2DM remains incomplete. Expansion of the availability of reliable biochemical markers in the blood could enhance this understanding and potentially lead to the identification of novel therapeutic targets to treat and prevent T2DM. At the same time, they may allow us to better predict the risk of T2DM and serve as biomarkers of the efficacy of measures taken to prevent diabetes. 
Sphingolipids (SPs), including ceramides, sphingomyelins, and cerebrosides, represent an abundant and structurally diverse class of lipids that are known to contribute to physiological and pathological processes (1). Notably, alterations in subclasses of SPs have been identified in T2DM patients and in animal models of diabetes. For example, ceramides have been shown to be elevated in the plasma of human T2DM patients (2), and studies of small cohorts suggested that there may be similar increases in plasma glucosylceramides $(3,4)$. In animal models, obese, diabetic mice were found to have total sphingomyelin and ceramide content that was decreased in adipose tissues but increased in plasma (5), whereas in wild-type mice, a high-fat diet resulted in increased ceramide in both adipose tissues and plasma (6). It is likely that there is functional significance to these changes, contributing to the pathology of metabolic disease or resulting from its sequelae. Specifically, in experimental animal models, multiple studies have demonstrated that ceramides promote the dysfunction and apoptosis of pancreatic beta islet cells in vitro and in vivo $(7,8)$ and that inhibition of ceramide synthesis improves insulin sensitivity (9). It is notable that these studies typically used fairly low-resolution analyses and evaluated broad categories of SPs without discrimination of specific SP molecules. Because each category is composed of many structural variants, it has been proposed that subclasses or individual species may be particularly relevant (10). For example, specific ceramides (18:0, or dihydroceramides), rather than total ceramide content, are selectively elevated before the onset of T2DM and thus may serve as a prognostic biomarker (11). Furthermore, deoxysphingolipids and d16:1 sphingosines were shown to be selectively elevated in metabolic syndrome and T2DM, respectively (12). These studies have been limited in their resolving power but underscore the need to evaluate detailed SP profiles.

Recent progress in mass spectrometry-based technologies allows for better discrimination of individual SP species than was previously possible (13-15). Such advances have expanded the repertoire of the "sphingolipidome" to over 600 structurally distinct SPs that have been confirmed and potentially thousands of theoretical metabolites that are likely to exist (1). Because SP metabolism is dysregulated in metabolic diseases, any of these specific molecules may represent a potential biomarker of T2DM or other pathologies (10). To date, there have been few reports of a population analysis of plasma SP content that have had the throughput and the resolution necessary to identify specific species of SPs as biomarker candidates. The current study was carried out to identify associations between SPs and parameters of obesity and diabetes in one of the largest population-based studies in an East Asian population to date.

\section{Results}

All 2302 participants (Figure 1) yielded quantifiable SP profiles in this study. The relative concentrations of the 80 structurally distinct, quantifiable lipid species (Supplemental Figures 1 and 2; supplemental material available online with this article; https://doi.org/10.1172/jci.insight.126925DS1) clustered in a pattern that was generally consistent with their metabolic pathways (Supplemental Figure 3). A notable exception involved the C16 ceramides (Cer d18:1/16:0 and Cer d18:2/16:0), which did not cluster with the other ceramides. This may be due to atypical regulation of a specific ceramide synthase that preferentially generates C16 ceramides, likely CERS6 (16).

Table 1 shows the characteristics of the study population as a whole and compares those who did and did not develop diabetes mellitus. Those who developed diabetes were older and had higher BMI, waist/ hip ratio, blood pressure, blood triglycerides, C-reactive protein, fasting insulin, and HbA1c. They also had lower levels of blood HDL and total- and low-molecular weight adiponectin.

The plasma concentrations of many SPs correlated with clinical and biochemical characteristics (Figure 2 and Figure 3). We first evaluated the associations between SPs and age and sex. SMs were generally more abundant in women, while SPHs were higher in men (Supplemental Figure 4 and Supplemental Table 1). Interestingly, complex SPs (SMs, HexCer, and Hex2Cer) containing a d18:2 sphingoid base preferentially demonstrated significantly higher levels in women. The SP classes most broadly associated with age were the ceramides, which largely increased with increasing age, and the hexosylceramides (HexCer and Hex2Cer), which almost uniformly decreased with increasing age (Figure 3, Supplemental Figure 5, and Supplemental Table 2).

Most of the risk factors for incident diabetes showed strong correlations with SPs. For example, body mass index (BMI) (Figure 4A), HOMA-IR (Figure 4B), waist-hip ratio (WHR), blood pressure, fasting insulin, C-reactive protein (CRP), and low levels of HDL and adiponectin all correlated positively with SPH, S1P, and most ceramides, but negatively with HexCer and Hex2Cer. SMs correlated positively or negatively depending on the molecular species. Among the ceramides, those with the noncanonical d16:1 backbone had more strongly positive correlations relative to the canonical d18:1 ceramides, while the d18:2 


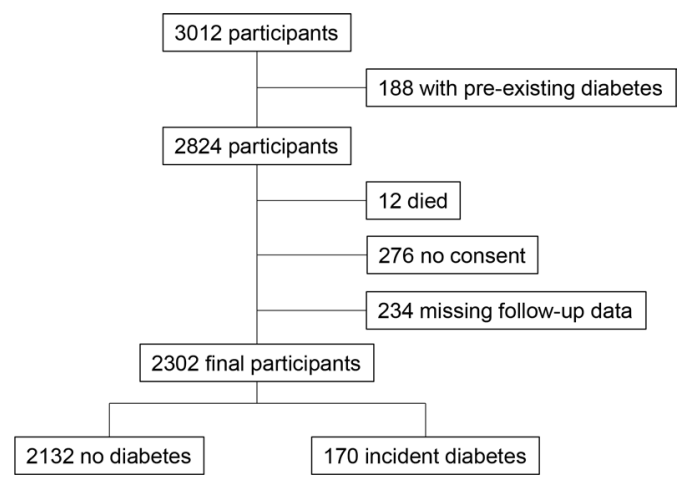

Figure 1. Flow diagram of the final number of participants included in the study.

ceramides correlated less positively or, in some cases, correlated negatively (Figure 3; Figure 4, A and B; Supplemental Table 3; and Supplemental Figures 6-8). In addition, the most strongly positive correlating ceramides were those containing a C18:0, C20:0, or C22:0 N-linked fatty acid. These phenomena were less consistent among the other, nonceramide, SP classes.

The strongest correlations were observed between LDL and most of the SPs, which likely reflects the fact that these SPs are carried on LDL in the plasma (Figure 3 and Supplemental Figure 6). Because experimental evidence demonstrates that the production of SPs increases with hyperlipidemia because of substrate availability (17) and that SPs make up a significant portion of LDL (18), these correlations were expected. In contrast, SPs correlated with HDL and triglycerides positively or negatively depending on the identity of the different SP species (Figure 3 and Supplemental Figures 7 and 8). Notably, HDL, which is known to transport SMs, correlated positively with most SMs but negatively with most ceramides, which are preferentially transported by LDL.

To determine the influence of these covariates on the relationship between SPs and BMI, we performed a multivariate analysis correcting for age, sex, LDL, HDL, and triglycerides (Figure 4C and Supplemental Tables 4 and 5). Most relationships were retained in this multivariate model. However, this analysis provided increased support for the significance of the noncanonical sphingoid backbone. That is, after correcting for these covariates, nearly all d16:1 SPs correlated positively and d18:2 SPs correlated negatively with BMI, regardless of the SP subtype.

The same SPs that were associated with BMI also showed a similar association with HOMA-IR. Given that some of these associations may be driven by the correlation between BMI and HOMA-IR, multivariate analyses were performed between SPs and HOMA-IR after correcting for age, sex, LDL, HDL, triglycerides, and BMI. Of the 70 SPs that correlated significantly with HOMA-IR, 38 remained significant in the multivariate model (Figure 4C and Supplemental Tables 4 and 5). Notably, these included all the SPHs and the d18:2 hexosylceramides (Figure 5). In contrast, most of the d18:1 SPs (70\%) that were significant in the univariate analysis became insignificant in the multivariate analysis, suggesting that these SPs correlated with insulin resistance as a consequence of their relationship with body mass and plasma lipids. Furthermore, most d18:2 Hex2Cers did not retain association with HOMA-IR after elastic net regularization (Supplemental Table 5), suggesting that d18:2 ceramides and d18:2 SMs are better predictors of the relationship between d18:2 SPs and HOMA-IR.

To determine whether SPs were significantly correlated with incidence of T2DM, a Cox regression analysis was performed to identify associations with follow-up diabetes incidence. Forty-nine SPs associated significantly with diabetes occurrence. Consistent with the correlation observed between SPs and $\mathrm{BMI}$, ceramides were generally higher in individuals who were subsequently diagnosed with T2DM, hexosylceramides were uniformly lower, and SMs varied by species (Figure 6 and Supplemental Tables 6-8). In addition, associations involving the d16:1 SPs were more strongly positive across SP classes relative to those with $\mathrm{d} 18: 1$ or $\mathrm{d} 18: 2$ sphingoid backbones. We then performed a multivariate Cox regression analysis to correct for diabetes-related covariates, including age, sex, LDL, HDL, triglycerides, BMI, and $\mathrm{HbA1c}$. As we observed when we examined the association between SPs and HOMA-IR, most of the associations were attenuated and became statistically nonsignificant after adjustment for BMI. Only 4 SPs, SM d16:1/18:0 and SM d18:1/18:0 and their metabolic precursors, Cer d16:1/18:0 and Cer $\mathrm{d} 18: 1 / 18: 0$, remained significant after adjustment for BMI, and when $\mathrm{HbA} 1 \mathrm{c}$ was added to the model, only the 2 SMs remained independently associated with T2DM incidence (Table 2). A net reclassification index (NRI) analysis demonstrated that the addition of SM d16:1/18:0 and SM d18:1/18:0 to the current clinical standard of $\mathrm{HbA} 1 \mathrm{c}$ or $\mathrm{HbA} 1 \mathrm{c}$ plus BMI provides moderately improved accuracy of 
Table 1. Clinical and demographic characteristics of study participants

\begin{tabular}{|c|c|c|c|c|c|c|}
\hline \multirow[t]{2}{*}{ Parameters } & \multicolumn{2}{|c|}{ Total population (2302 subjects) } & \multicolumn{2}{|c|}{ No diabetes (2132 subjects) } & \multicolumn{2}{|c|}{ Incident diabetes (170 subjects) } \\
\hline & Median & Interquartile range & Median & Interquartile range & Median & Interquartile range \\
\hline BMI $\left(\mathrm{kg} / \mathrm{m}^{2}\right)$ & 22.32 & $20.18-24.74$ & 22.15 & 20.04-24.46 & 24.76 & $22.35-27.55$ \\
\hline HbA1c (\%) & 5.39 & $5.20-5.69$ & 5.39 & $5.20-5.69$ & 5.89 & $5.59-6.09$ \\
\hline Fasting insulin $(\mu \mathrm{U} / \mathrm{mL})$ & 5.40 & $3.80-8.00$ & 5.30 & $3.70-7.60$ & 8.60 & $6.03-12.30$ \\
\hline Glucose (nmol/L) & 4.70 & $4.40-5.00$ & 4.60 & $4.40-4.90$ & 5.10 & $4.70-5.50$ \\
\hline Total cholesterol (mmol/L) & 5.15 & $4.60-5.79$ & 5.14 & $4.58-5.78$ & 5.34 & $4.72-6.03$ \\
\hline HDL-cholesterol (mmol/L) & 1.45 & $1.24-1.71$ & 1.46 & $1.24-1.72$ & 1.30 & $1.11-1.52$ \\
\hline LDL-cholesterol (mmol/L) & 3.09 & $2.60-3.67$ & 3.08 & $2.59-3.66$ & 3.16 & $2.64-3.87$ \\
\hline Systolic BP (mmHg) & 126.50 & $114.50-141.50$ & 125.00 & $114.00-140.50$ & 143.50 & $130.75-158.38$ \\
\hline Diastolic BP (mmHg) & 75.50 & $68.00-84.00$ & 75.00 & $68.00-84.00$ & 82.00 & $74.63-89.50$ \\
\hline
\end{tabular}

Among the 2302 study subjects, there were 1056 men and 1246 women. HOMA-IR, homeostatic model assessment of insulin resistance; CRP, C-reactive protein; WHR, waist/hip ratio.

the prediction of diabetes incidence for $38 \%-40 \%$ of individuals (Supplemental Table 9). For individuals that became diabetic, predicted probability was increased in $59 \%-61 \%$ and decreased in $39 \%-41 \%$, giving an $\mathrm{NRI}^{+}$of $18 \%-21 \%$. For individuals who did not become diabetic, predicted probability was reduced in $59 \%-60 \%$ and increased in $40 \%-41 \%$, giving an $\mathrm{NRI}^{-}$of $18 \%-20 \%$. Similar results were obtained when evaluating the effect of adding SM d16:1/18:0 and SM d18:1/18:0 to the Atherosclerosis Risk in Communities predictive model for diabetes risk (ref. 19 and Supplemental Table 9). Interestingly, the improvements of these models were entirely due to SM d16:1/18:0, while SM d18:1/18:0 alone did not alter the models beyond a $95 \%$ CI. It is also of note that although the addition of SM d16:1/18:0 did improve the prediction of future diabetes risk for a relatively large proportion of the population, the magnitude of change of the predicted probability was modest (Supplemental Figure 9) and, as a consequence, may not have a large effect at the population level despite the improvement in prediction.

\section{Discussion}

To the best of our knowledge, this study reports the largest population-based, high-resolution analysis of the sphingolipidome, allowing for an accurate evaluation of the associations between plasma SP content and obesity- and diabetes-related characteristics, such as BMI, blood lipids, and HOMA-IR. Many of the SPs evaluated in this study demonstrated significant correlations with BMI. Surprisingly, these correlations were not uniformly positive, which would be expected if SP synthesis were globally upregulated with increasing lipid stores. Rather, these correlations suggest that a more complex derangement of SP metabolism results from increased adiposity and/or contributes to susceptibility to obesity.

Consistent with the known diabetogenic role of ceramides (7), we found that most ceramides correlated positively with HOMA-IR and other diabetes-related characteristics. Interestingly, this included an inverse relationship between ceramides and adiponectin. Although this may be due to the known negative correlation between this adipokine and BMI (20), there may be a more direct relationship between adiponectin and ceramide. Notably, adiponectin administration has been shown to induce ceramidase activity in vitro and in vivo, via endogenous ceramidase activity of the adiponectin receptor $(21,22)$. Indeed, most ceramides retained significant positive associations with adiponectin even with the use of BMI as a covariate (Supplemental Table 10).

In contrast with the positive relationship between most ceramides and diabetes-related characteristics, our study found a significant negative correlation between hexosylceramides and diabetes-related characteristics. These results suggest that there is an increase in the scavenge/salvage pathway in obese individuals that leads to the production of ceramides through the catabolism of hexosylceramides (Figure 7). 


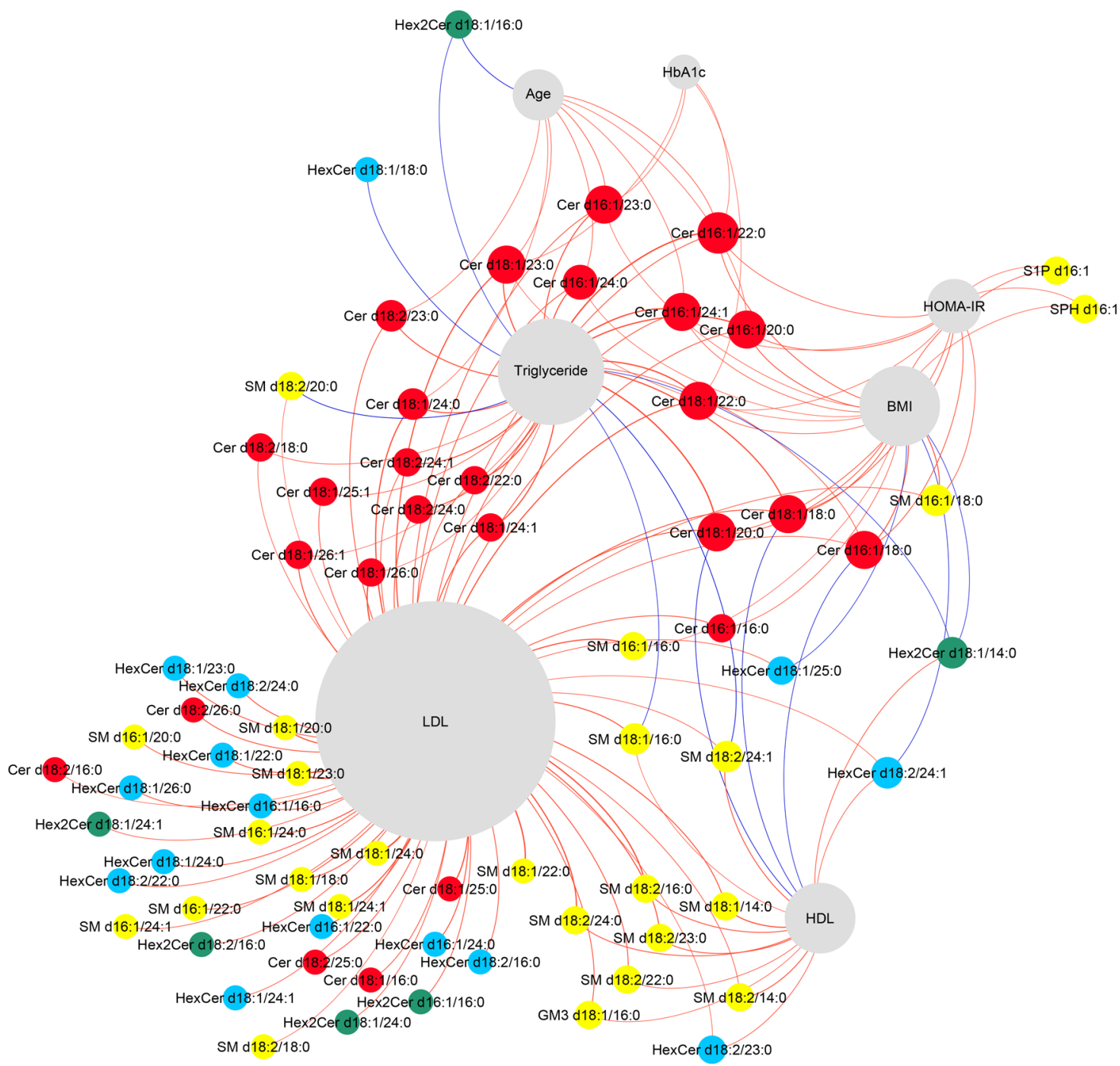

Figure 2. Correlations between SPs and key clinical characteristics. SP/clinical characteristics network showing plasma SP correlations with 7 key clinical characteristics. Pearson's correlation coefficients based on univariate logistic regression are represented as connecting lines (edges) between the clinical characteristics' nodes and SPs' nodes. Only correlations with an absolute value of 0.2 and above were shown. Edge width is proportional to correlation strength from minimum 0.2 to maximum 0.58. Size of circles (nodes) is proportional to degree (total number of connections with other nodes). Red edge color indicates positive correlation while blue edge indicates negative correlation. Node color was grouped according to SP type and clinical characteristic: clinical characteristic nodes are gray, Cer nodes are red, HexCer nodes are blue, Hex2Cer nodes are green, and nodes for the other SPs are yellow. The graph was generated using the Force Atlas layout in Gephi 0.9.2. S1P, sphingosine-1-phosphate; SM, sphingomyelin; SPH, sphingosine.

We found that plasma hexosylceramide content associated negatively with both BMI and T2DM incidence, which seems to contradict previous preclinical studies that have suggested that hexosylceramides are likely to be negative regulators of insulin signaling. For example, inhibition of hexosylceramide metabolism in mice and rats was associated with improved glucose tolerance and insulin sensitivity $(23,24)$. Furthermore, concentrations of ganglioside GM3 were found to be increased in the adipose tissue of obese, insulin-resistant women (25), and a negative association was found between muscle ganglioside GM3 concentration and insulin sensitivity (26). Rats fed a high-fat, obesogenic diet for 12 weeks had an accumulation of hexosylceramides in adipose tissue but a depletion in muscle (27). However, in rodent diabetes models, it was found that muscle hexosylceramide levels increased in a T2DM model but decreased in a T1DM model (28). In the current study, we identified only 1 GM3 species (d18:1/16:0). Although this lipid was negatively associated with BMI and HOMA-IR, and it correlated closely with its metabolic precursor, Hex2Cer d18:1/16:0, it is possible that other, more complex ceramides are differentially regulated. This is consistent with in vitro studies that found distinguishable effects of ceramides versus hexosylceramides on insulin signaling (27). 
$\sum_{\substack{1 \\ \sum_{i}}}^{3}$

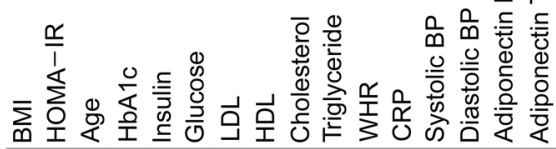

Cer d16:1/16:0

Cer d16:1/18:0

Cer d16:1/20:0

Cer d16:1/22:0

Cer d16:1/23:0

Cer d16:1/24:0

Cer d16:1/24:1

Cer d18:1/16:0

Cer d18:1/18:0

Cer d18:1/20:0

Cer d18:1/22:0

Cer d18:1/23:0

Cer d $18: 1 / 24: 0$

Cer d18:1/24:1

Cer d18:1/25:0

Cer d18:1/25:1

Cer d18:1/26:0

Cer d18:1/26:1

Cer d18:2/16:0

Cer d18:2/18:0

Cer d $18 \cdot 2 / 22 \cdot 0$

Cer d18:2/23:0

Cer d18:2/24:0

Cer d18:2/24:1

Cer d $18 \cdot 2 / 25 \cdot 0$

Cer d 18:2/26:0

Hex2Cer d16:1/16:0

Hex2Cer d18:1/14:0

Hex2Cer d18:1/16:0

Hex2Cer d18:1/24:0

Hex2Cer d18:1/24:1

Hex2Cer d18:2/16:0

GM3 d18:1/16:0

HexCer d16:1/16:0

HexCer d16:1/22:0

HexCer d16:1/24:0

HexCer d18:1/16:0

HexCer d18:1/18:0

HexCer d18:1/22:0

HexCer d18:1/23:0

HexCer d18:1/24:0

HexCer d18:1/24:1

HexCer d18:1/25:0

HexCer d18:1/26:0

HexCer d18:2/16:0

HexCer d18:2/22:0

HexCer d18:2/23:0

HexCer d 18:2/24:0

HexCer d18:2/24:1

SM d16:1/16:0

SM d16:1/18:0

SM d16:1/20.0

SM d16:1/22:0

SM d16:1/24:0

SM d16:1/24:1

SM d18:1/14:0

SM d18:1/16:0

SM d18:1/18:0

SM d18:1/20:0

SM d18:1/22.0

SM d18:1/23:0

SM d18:1/24:0

SM d18:1/24:1

SM d18:2/14:0

SM d18:2/16:0

SM d18.2/18.0

SM d18.2/20.0

SM d18:2/22:0

SM d18:2/23:0

SM d18:2/24:0

SM d18:2/24:1

SPH d16:1

SPH d18:0

SPH d18:1

SPH d18:2

S1P d16:1

S1P d17:1

S1P d18:0

S1P d18:1

S1P d18:2
Figure 3. Correlations between SPs and clinical characteristics. Pearson's correlation coefficients based on univariate logistic regression were calculated between each SP species and relevant clinical characteristics and depicted as a heatmap. Positive correlations are depicted in red. Negative correlations are depicted in blue. 
A

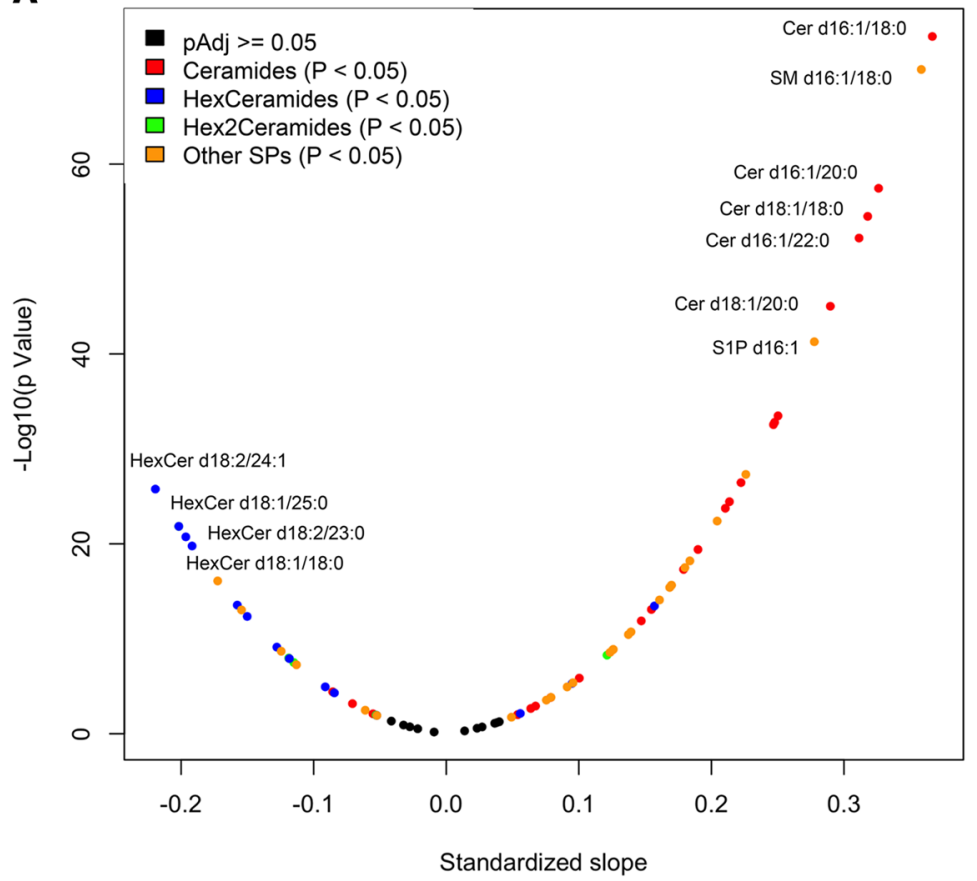

B

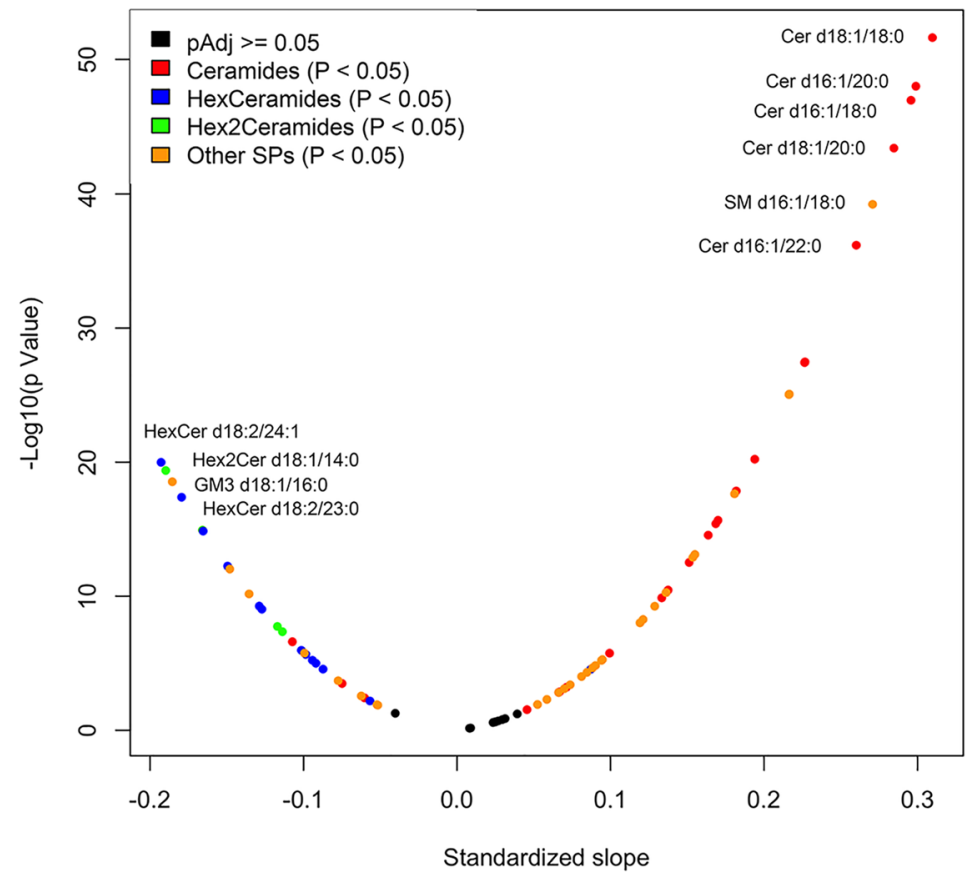

C

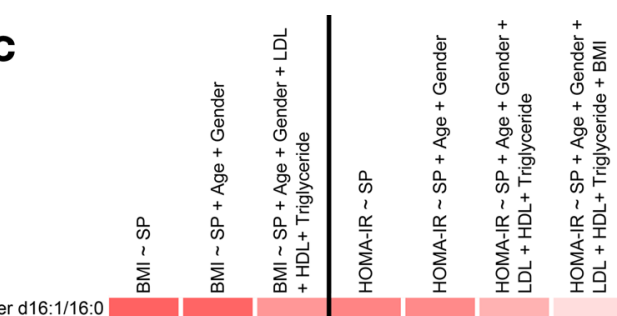

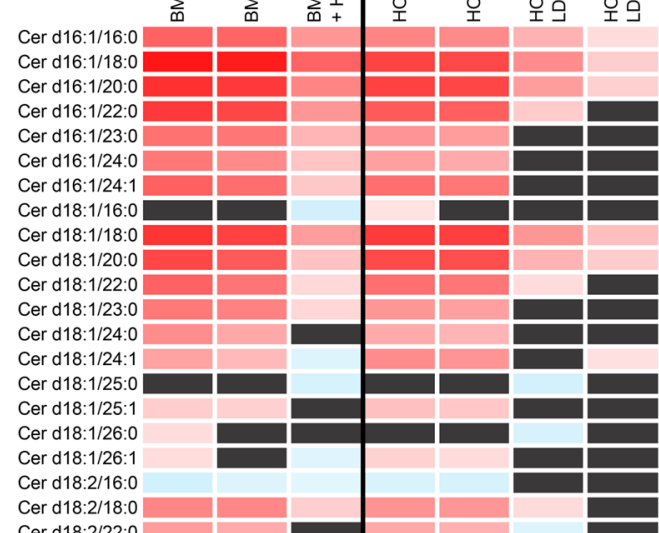

Cer d18:2/22:0

Cer d18:2/23:0

Cer d18:2/24:0

Cer d18:2/24:1

Cer d18:2/25:0

Cer d18:2/26:0

Hex2Cer d16:1/16:0

Hex2Cer d18:1/14:0

Hex2Cer d18:1/16:0

Hex2Cer d18:1/24:0

Hex2Cer d18:1/24:1

Hex2Cer d18:2/16:0

GM3 d18:1/16:0

HexCer d16:1/16:0

HexCer d16:1/22:0

HexCer d16:1/24:0

HexCer d18:1/16:0

HexCer d18:1/18:0

HexCer d18:1/22:0

HexCer d18:1/23:0

HexCer d18:1/24:0

HexCer d18:1/24:1

HexCer d18:1/25:0

HexCer d18:1/26:0

HexCer d18:2/16:0

HexCer d18:2/22:0

HexCer d18:2/23:0

HexCer d18:2/24:0

HexCer d18:2/24:1

SM d16:1/16:0

SM d16:1/18:0

SM d16:1/20:0

SM d16:1/22:0

SM d16:1/24:0

SM d16:1/24:1

SM d18:1/14:0

SM d18:1/16:0

SM d18:1/18:0

SM d18:1/20:0

SM d18:1/22:0

SM d18:1/23:0

SM d18:1/24:0

SM d18:1/24:1

SM d18:2/14:0

SM 118.2/16:0

SM d18:2/18:0

SM d18:2/20:0

SM d18:2/22:0

SM d18:2/23:0

SM d18:2/24:0

SM d18:2/24:1

SPH d16:1

SPH d18:0

SPH d18:1

SPH d18:2

S1P d16:1

S1P d17:1

S1P d18:0

S1P d18:1

S1P d18:2 
Figure 4. Linear regression analysis between SPs and BMI and HOMA-IR. Volcano plots of the effects of (A) BMI on lipid concentration representing the normalized slope of the lipid/BMI relationship versus the significance of this relationship and (B) HOMA-IR on lipid concentration representing the normalized slope of the lipid/HOMA-IR relationship versus the significance of this relationship. Lipids are color coded by class, except that those without a significant $(P>0.05)$ relationship are indicated in black. (C) Multivariate linear regression analysis between SPs and BMI and HOMA-IR. Models with increasing covariates are depicted from left to right (increasing stringency). The shading intensity is proportional to the slope of the regression line. Relationships that are not significant $(P>0.05)$ are indicated with a black box.

In addition to the identification of an inverse association between plasma hexosylceramides and T2DM, this study identified unexpected correlations between increased BMI and SPs that contain d16:1 and d18:2 sphingoid backbone structures. These noncanonical SPs represent a quantitatively minor component of the sphingolipidome, relative to d18:1 SPs, which may explain why this relationship was underappreciated in previous studies. With respect to their correlations with BMI and HOMA-IR, d16:1 SPs are uniformly higher and d18:2 SPs are often lower than that of the canonical d18:1 species. This pattern is consistent across subclasses, including ceramides, hexosylceramides, SPHs, and S1P.

SP chain length is regulated at the first step of de novo synthesis by a heterotrimeric SPT enzyme complex (29). SPT long chain base unit 1 (SPTLC1) is an obligate member of this complex, but differential incorporation of 1 of 2 additional large subunits (SPTLC2 or SPTLC3) and 1 of 2 small subunits (SPT small subunit A [SPTSSA] or SPT small subunit B [SPTSSB]) results in alterations in substrate preference (29, 30). Therefore, it is likely that accumulation of d16:1 SPs is due to a dysregulation of SPT subunit genes as a result of increased adiposity. The key SPT subunit involved in the generation of SPs with shorter backbones is SPTLC3, which catalyzes the formation of d16:1 SPH (29). Interestingly, SPTLC3 has been shown to generate $\mathrm{d} 16: 1$ SPs in the heart, where high levels of d16:1 SPs were found to induce cardiomyocyte apoptosis (29, 31 ), thus identifying a potential mechanistic link between T2DM and congestive heart failure. Alternatively, dysregulation of SPT small subunits may be contributing to this phenomenon because SPTSSA has been shown to exhibit greater preference for shorter acyl chain substrates than those of SSPTSSB (30).

The origin of d18:2 SPs (with a second double bond in the $14 \mathrm{Z}$ position) is less clear because there are no known metabolic pathways that are responsible for their generation (1). This may be due to the incorporation of monounsaturated fatty acids by SPT; however, the likely precursor, 12-hexadecenoic acid, is not detected in human plasma in significant concentrations (32). Alternatively, it is possible that an unknown SP desaturase catalyzes the incorporation of a second double bond into the sphingoid backbone at some step along the SP metabolic pathway (33).

We also observed asymmetries in the correlations relating to ceramides with different N-linked fatty acids (Figure 2 and Figure 3). Notably, medium-chain ceramides (C18:0, C20:0, and C22:0) correlated more positively with BMI, HOMA-IR, and diabetes incidence than other ceramides, regardless of the nature of the sphingoid backbone. This may be due to alterations in the expression or activity of specific ceramide synthase genes, such as CERS1 and CERS4, which are known to generate these ceramide species preferentially $(34,35)$. Furthermore, it is likely that this has functional significance because generation of these ceramides by CERS4 is associated with glucolipotoxicity in pancreatic $\beta$ cells in vitro (34).

It is likely that these observed trends in the levels of the noncanonical subclasses of SPs (considering specific head groups, sphingoid backbones, and $\mathrm{N}$-acyl fatty acids) are the result of broad, consistent shifts in 3 key metabolic steps: SPT-mediated condensation (sphingoid backbone variation), CERS-mediated N-acetylation (fatty acid length), and hexosyltransferase activity (HexCer accumulation). Such activity should result in broad, consistent changes in all SPs of that subtype, rather than sporadic changes in a few species. Indeed, when considering the subclasses in aggregate by multivariate linear regression analysis, hexosylceramides remained negatively associated with BMI, while SPs and d16:1 SPs (but not d18:1 SPs) containing very longchain fatty acids remained positively associated with BMI (Supplemental Figure 10). In contrast, these relationships did not retain association with T2DM incidence by Cox regression analysis when adjusted for age, sex, LDL, HDL, triglycerides, BMI, and HbA1c (Supplemental Figure 11). This is consistent with the high stringency of this analysis and emphasizes the importance of obtaining high-resolution lipidomic profiles.

Interestingly, while this manuscript was in preparation, 2 additional population-based lipidomics analyses evaluated plasma SPs in the context of obesity and diabetes $(36,37)$. Relative to the study presented here, the Huynh et al. study (36) quantified a larger number of metabolites (636 species), across more lipid classes (SPs, phospholipids, cholesterols, acylcarnitines), in fewer individuals (640 samples), whereas the Lemaitre et al. study (37) evaluated fewer SPs (15 species) on a similar number of individuals (2086 sam- 


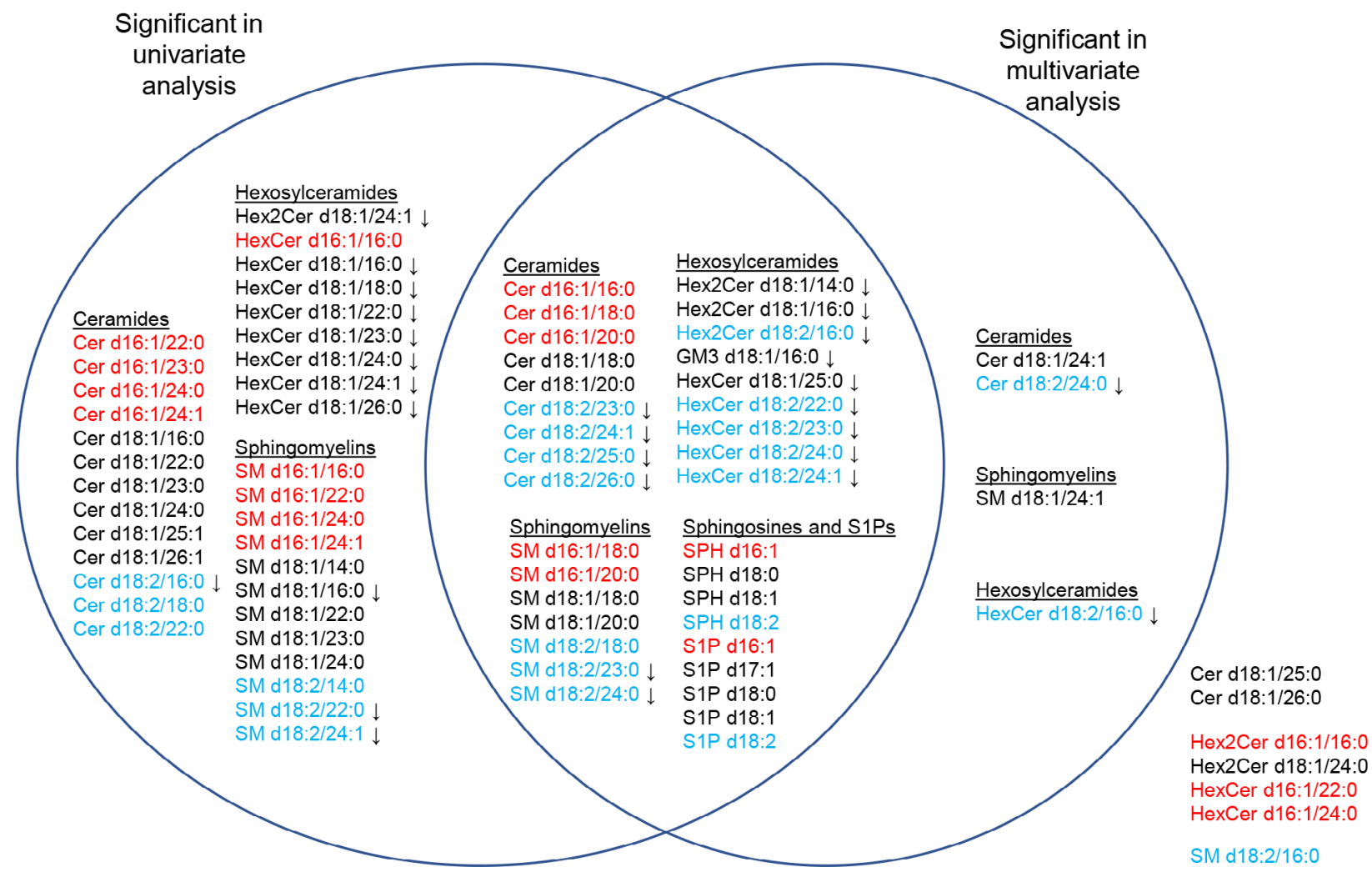

Figure 5. Venn diagram depicting SP species that significantly correlate with HOMA-IR under different linear regression models. SPs with a d16:1 backbone are indicated in red. SPs with a d18:2 backbone are indicated in blue. All relationships are positive unless indicated with " $\downarrow$ ".

ples). Although there were some differences among our 3 studies; interestingly, 1 significant observation was consistent: ceramides correlated positively while hexosylceramides correlated negatively with clinical and biochemical characteristics of obesity and diabetes. This is particularly notable because these studies evaluated 3 ethnically distinct cohorts, Australian, Native American, and Singaporean Chinese, suggesting that our observations are not specific to an East Asian population. It is notable that these studies were performed with different analytical methodologies, which is known to result in some variation in absolute lipid concentrations (38). However, the relative concentrations determined in the current study were consistent with those reported by Huynh et al. (Supplemental Figure 12), and the commonalities reported among these studies independently validated some of our major findings. Neither of the recent studies evaluated longitudinal diabetes incidence data; however, Huynh et al. (36) demonstrated a significant correlation between 1 of our putative prognostic markers, SM d18:1/18:0, and fasting glucose. (SM d16:1/18:0 was not evaluated.) Interestingly, Lemaitre et al. (37) reported a complex relationship between several SMs (including SM d18:1/18:0) and insulin sensitivity that was dependent upon BMI. That is, SMs are negatively associated with insulin level at normal BMI but are positively associated in obese individuals.

Although factors such as lifestyle and elevated BMI may predispose individuals to increased risk, there remains an absence of specific prognostic biomarkers for progression to T2DM. Our results demonstrate that BMI and HOMA-IR correlated positively with d16:1 SPs and correlated negatively with d18:2 SPs and with hexosylceramides. Furthermore, our survival analysis found that 2 SPs (SM d16:1/18:0 and SM d18:1/18:0) were significantly associated with diabetes occurrence, independent of age, sex, plasma lipid profile, and BMI, with HRs of about 1.4, suggesting that these SPs may be used as potential novel predictors for the development of diabetes.

Limitations of the study. Because the participants of this study were entirely of East Asian ethnicity, it is not clear whether all the findings presented here can be generalized to an ethnically diverse population. For example, a previous study found that total SM levels were higher in Asian Americans than in Americans with European ancestry (39). A study of the biological variation of the lipidome across the different ethnic groups (Malay, Chinese, and Indian groups) in Singapore also showed specific associations between several 


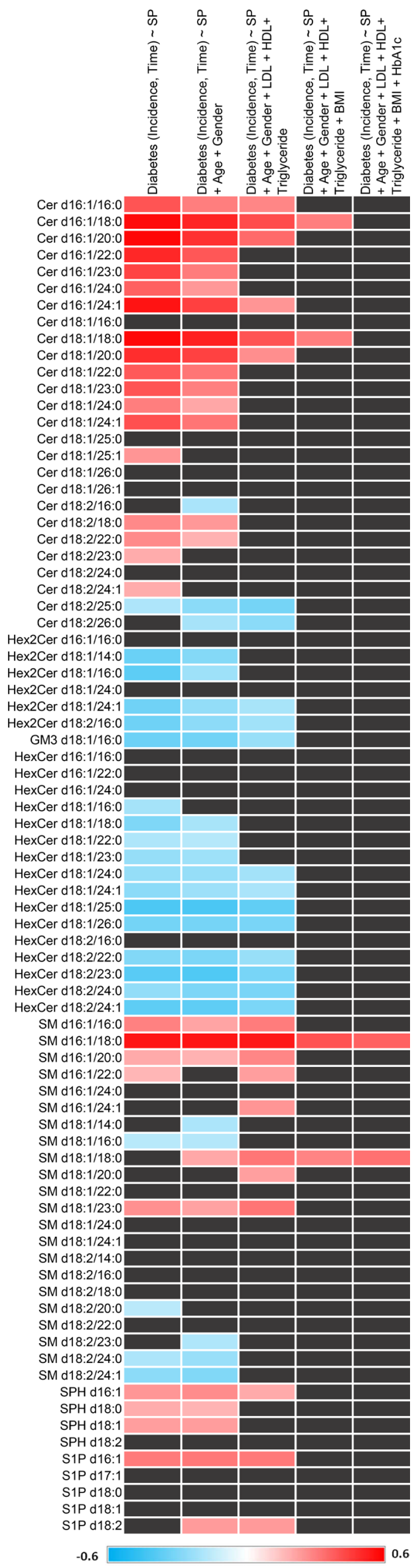

Figure 6. Cox regression analysis between baseline SP concentrations and subsequent diagnosis of T2DM. Models with increasing covariates are depicted from left to right (increasing stringency). The shading intensity is proportional to the slope of the regression line. Relationships that are not statistically significant $(P>0.05)$ are indicated with a black box. 
Table 2. Cox regression analysis and HR of the effect of baseline SP concentration on subsequent diabetes incidence after adjusting for age, sex, LDL, HDL, triglycerides, BMI, and HbA1c

\begin{tabular}{lccr}
\hline Lipid & Adjusted $\boldsymbol{P}$ value & HR & $\mathbf{9 5 \%}$ Cl \\
Cer d16:1/18:0 & 0.40 & 1.24 & $1.01-1.52$ \\
Cer d18:1/18:0 & 0.12 & 1.35 & $1.09-1.67$ \\
SM d16:1/18:0 & $\mathbf{1 . 4 7 E - 0 2}$ & 1.45 & $1.18-1.78$ \\
SM d18:1/18:0 & $\mathbf{1 . 4 7 E - 0 2}$ & 1.40 & $1.17-1.68$
\end{tabular}

Significant associations $(P<0.05)$ are indicated in bold.

lipid species and certain ethnic groups (40). This suggests that there are genetic factors that regulate basal SP metabolism (41) and that resulting differences may explain ethnic variability in disease susceptibility and other similar lipidomic studies.

This study involved the evaluation of SPs in plasma only. Although this is ideal for the identification of systemic biomarkers, there are limitations for the mechanistic implications of the resulting data. Notably, plasma levels may not correlate with the effective concentrations of SPs in target tissues, such as liver, muscle, and adipose. This may account for some apparent paradoxes. For example, the negative correlations between plasma hexosylceramides and HOMA-IR may reflect sequestration of these SPs from the plasma into muscle and adipose, rather than being the result of global hexosylceramide depletion. This would explain the disparity between this study and others that suggest a positive relationship between hexosylceramides and insulin resistance.

\section{Methods}

Study population. Participants were recruited between 2004 and 2007 as part of the Singapore prospective study program and the Singapore cardiovascular cohort study as described previously (42). Briefly, the participants were interviewed in their homes and invited to undergo a health examination for additional tests and collection of biological specimens at local hospitals. Age, sex, height, weight, and blood pressure were determined during the in-home interview, as described previously (42). Baseline plasma samples were obtained from participants after an 8- to 12-hour fast (42), from which LDL, HDL, triglycerides, and $\mathrm{HbA1c}$ levels were determined using standard enzymatic assays (43). All SP measurements were per-

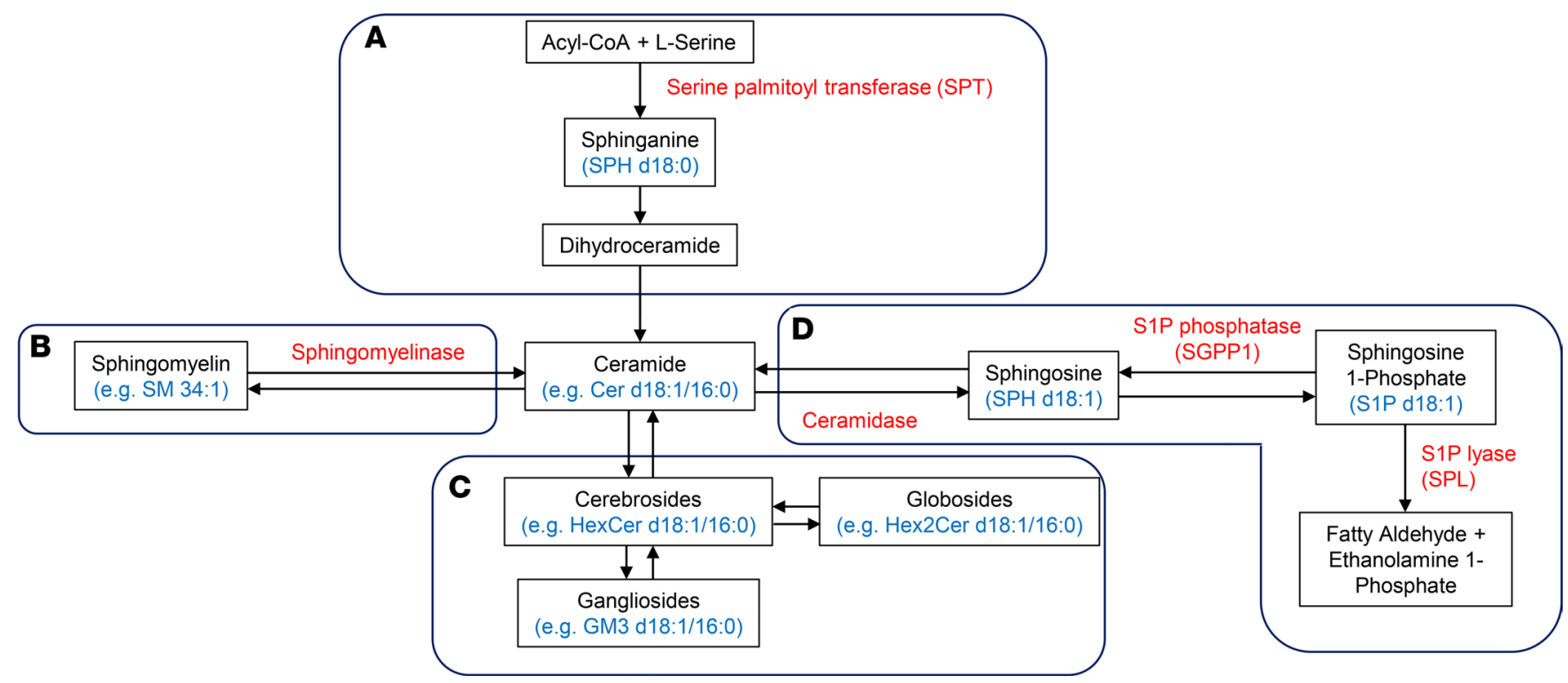

Figure 7. SP metabolism. This schematic shows a simplified representation of the SP metabolic pathway. (A) De novo synthesis results in the formation of the essential sphingoid backbone with the condensation of serine and a fatty acyl CoA by SPT. (B) The sphingomyelinase pathway generates ceramides from the catabolism of membrane stores of SM. (C) Ceramides are glycosylated into complex ceramides (including HexCer and Hex2Cer) and can be recycled back to ceramides through the scavenge/salvage pathway. (D) The degradation pathway terminates with the irreversible catabolism of S1P by SPL. The resulting products may enter the Kennedy pathway for the de novo synthesis of membrane phospholipids. 
formed on baseline plasma samples. Because of the known variation in SP profiles among the different ethnic groups within Singapore (44), we excluded non-Chinese participants. In addition, participants with diabetes at baseline, participants who had died, and participants who did not provide consent for their diabetes status to be tracked were excluded, resulting in a total of 2302 individuals with health screening data and matched plasma samples that were analyzed in this study (Figure 1 and Table 1).

Assessment of incident diabetes. Participants were evaluated with periodic follow-up visits for approximately 11 years after initial sample collection and their diabetes status was assessed. Assessment of participants' diabetes status was dependent on diabetes diagnosis detected by a nationwide governmental database covering public health care systems or participants' self-reported physician diagnosis based on 1 or more of the following criteria: fasting plasma glucose $(\geq 7.0 \mathrm{mmol} / \mathrm{L})$ or $\mathrm{HbAlc}(\geq 48 \mathrm{mmol} / \mathrm{mol})$ or concentrations above the American Diabetes Association cutoffs for clinical diabetes. Using these criteria, 170 subjects were diagnosed with T2DM during the follow-up period (Table 1). The earliest date of diabetes diagnosis was then linked with the initial study interview and blood collection date to calculate time to event for subsequent data analyses. If a participant was identified as developing diabetes based on more than 1 criterion and therefore had 2 or more different dates of diagnosis available, the earliest date was used to determine time to event.

Lipid extraction and quantification. Lipids from plasma samples were extracted using a 1-butanol/methanol $(1: 1, \mathrm{v} / \mathrm{v})$ extraction solvent containing a set of internal standards based on a method previously described (45). 1-butanol and methanol were supplied by Merck Millipore. For S1P analysis, the extracted lipids underwent an additional trimethylsilyl-diazomethane derivatization step (14). The extracted lipids were analyzed by positive mode electrospray ionization mass spectrometry using an Agilent 6495 QQQ mass spectrometer. Lipid separations were performed on a UHPLC Agilent 1290, using a Waters BEH HILIC column (for S1P) or a reversed-phase Agilent ZORBAX Rapid Resolution High Definition Eclipse Plus C18 column (for all other SPs). Lipids were quantified using a dynamic, multiple reaction monitoring method with measurement of peak area of quantifier transitions by peak integration. Lipid peaks were identified based on their specific precursor and product ion transitions in addition to their retention time (45). Subsequent normalization with internal standards was carried out as previously discussed (45). To ensure the quality and precision of the results, pooled quality control (QC) samples were included every 10 study samples. The coefficient of variation $(\mathrm{CoV})$ of each individual lipid in the QC samples was then calculated, and lipids with $\mathrm{CoV}$ higher than 30\% were excluded. Evaluation was performed for 331 peaks for each plasma sample. Of these, 80 peaks met our inclusion criteria for reliability and were included in subsequent analyses. These included members of the following classes: ceramides, cerebrosides (monohexosylceramides and HexCer), globosides (dihexosylceramides and Hex2Cer), gangliosides (GM3), SMs, S1P, and SPHs (Supplemental Table 1). Only a small number of internal standards were used for this analysis, as is often the case for lipidomic measurements. We based our considerations on relative quantification of the endogenous species because absolute quantification would require either the use of a specific standard for each molecule measured or the evaluation of other factors, such as changes in matrix effects during elution.

Statistics. Principal component analysis plots of the relative abundance of the lipids in the samples and QCs were created to detect any potential drifts and batch effects. All analyses were performed using R (version 3.5.1, GNU General Public License v2) on a 64-bit Linux system. All values were log-transformed for analyses. Associations between BMI and SPs were evaluated using linear models (lm function) fitted to before and after adjusting for age, sex, LDL, HDL, and triglycerides. Similarly, linear models were also used to evaluate the associations between HOMA-IR and SPs after adjusting for age, sex, LDL, HDL, triglycerides, and BMI. Cox regression analysis (surv function) was also carried out to identify potential SPs associated with the risk of progression to T2DM after adjusting for age, sex, LDL, HDL, triglycerides, BMI, and HbA1c. Standardized slope (reghelper package) was calculated for all the models. $P$ values were adjusted by Benjamini-Hochberg method. Relationships were considered significant if the adjusted $P$ value was less than or equal to 0.05 .

Study approval. All human plasma samples were collected in accordance to ethical guidelines and protocols. This study was approved by the SingHealth Institutional Review Board (Centralised Institutional Review Board 2001/001/C), Singapore. Informed consent was obtained from all subjects.

\section{Author contributions}

WSC, FT, SJ, HB, MRW, EST, and DRH were involved in the study design. WSC and FT conducted the lipidomics experiments. XS and EST provided the plasma samples. HC, XS, RMVD, and EST provided the clinical and diabetes data. WSC, FT, SJ, HC, and DRH were involved in the statistical analysis of the data. 
CMK, EYHK, and WYO contributed advice and expertise on the discussion of the results. WSC and DRH wrote the manuscript. All authors contributed critical additions and revisions to the manuscript.

\section{Acknowledgments}

This work was supported by the National University Health System (NUHSRO/2014/085/AF-Partner/01, to DRH), the Ministry of Education, Singapore (T1- 2016 Sep -11 to DRH, and T1-2017 Oct -15 to WYO), and the National Research Foundation Investigatorship grant (NRF-NRFI2015-05, to MRW).

Address correspondence to: Deron R. Herr, National University of Singapore, Department of Pharmacology, 16 Medical Drive, MD3-04-01W, Singapore 117600, Singapore. Phone: 65.6516.6825; Email: phcdrh@ nus. edu.sg. Or to: E. Shyong Tai, National University of Singapore, Department of Medicine, 1E, Kent Ridge Road, NUHS Tower Block, Level 10, Singapore 119228, Singapore. Phone: 65.6772.4362; Email: mdctes@ nus.edu.sg. Or to: Markus R. Wenk, National University of Singapore, Department of Biochemistry, 8 Medical Drive, MD7, Singapore, 117596, Singapore. Phone: 65.6516.3682; Email: bchmrw@nus.edu.sg.

HB's present address is: Australian Breast Device Registry, Monash University, Melbourne, Australia.

1. Lai MK, et al. Biological effects of naturally occurring sphingolipids, uncommon variants, and their analogs. Neuromolecular Med. 2016;18(3):396-414.

2. Haus JM, et al. Plasma ceramides are elevated in obese subjects with type 2 diabetes and correlate with the severity of insulin resistance. Diabetes. 2009;58(2):337-343.

3. Kremer GJ, Atzpodien W, Schnellbacher E. Plasma glycosphingolipids in diabetics and normals. Klin Wochenschr. 1975;53(13):637-638.

4. Serlie MJ, et al. Short-term manipulation of plasma free fatty acids does not change skeletal muscle concentrations of ceramide and glucosylceramide in lean and overweight subjects. J Clin Endocrinol Metab. 2007;92(4):1524-1529.

5. Samad F, Hester KD, Yang G, Hannun YA, Bielawski J. Altered adipose and plasma sphingolipid metabolism in obesity: a potential mechanism for cardiovascular and metabolic risk. Diabetes. 2006;55(9):2579-2587.

6. Shah C, Yang G, Lee I, Bielawski J, Hannun YA, Samad F. Protection from high fat diet-induced increase in ceramide in mice lacking plasminogen activator inhibitor 1. J Biol Chem. 2008;283(20):13538-13548.

7. Chavez JA, Summers SA. A ceramide-centric view of insulin resistance. Cell Metab. 2012;15(5):585-594.

8. Aerts JM, et al. Glycosphingolipids and insulin resistance. Adv Exp Med Biol. 2011;721:99-119.

9. Chavez JA, et al. A role for ceramide, but not diacylglycerol, in the antagonism of insulin signal transduction by saturated fatty acids. J Biol Chem. 2003;278(12):10297-10303.

10. Chong JR, et al. Sphingolipidomics analysis of large clinical cohorts. Part 2: Potential impact and applications. Biochem Biophys Res Commun. 2018;504(3):602-607.

11. Wigger L, et al. Plasma dihydroceramides are diabetes susceptibility biomarker candidates in mice and humans. Cell Rep. 2017;18(9):2269-2279.

12. Othman A, et al. Plasma deoxysphingolipids: a novel class of biomarkers for the metabolic syndrome? Diabetologia. 2012;55(2):421-431

13. Bowden JA, et al. Harmonizing lipidomics: NIST interlaboratory comparison exercise for lipidomics using SRM 1950-Metabolites in Frozen Human Plasma. J Lipid Res. 2017;58(12):2275-2288.

14. Narayanaswamy P, et al. Lipidomic "deep profiling": an enhanced workflow to reveal new molecular species of signaling lipids. Anal Chem. 2014;86(6):3043-3047.

15. Chew WS, et al. Sphingolipidomics analysis of large clinical cohorts. Part 1: technical notes and practical considerations. Biochem Biophys Res Commun. 2018;504(3):596-601.

16. Mullen TD, Hannun YA, Obeid LM. Ceramide synthases at the centre of sphingolipid metabolism and biology. Biochem J. 2012;441(3):789-802.

17. Russo SB, Ross JS, Cowart LA. Sphingolipids in obesity, type 2 diabetes, and metabolic disease. In: Gulbins E, Petrache I, eds. Sphingolipids in Disease. Vienna, Austria: Springer Vienna; 2013:373-401.

18. Boon J, et al. Ceramides contained in LDL are elevated in type 2 diabetes and promote inflammation and skeletal muscle insulin resistance. Diabetes. 2013;62(2):401-410.

19. Chin CW, et al. The ARIC predictive model reliably predicted risk of type II diabetes in Asian populations. BMC Med Res Methodol. 2012;12:48.

20. Arita Y, et al. Paradoxical decrease of an adipose-specific protein, adiponectin, in obesity. Biochem Biophys Res Commun. 1999;257(1):79-83.

21. Holland WL, et al. Receptor-mediated activation of ceramidase activity initiates the pleiotropic actions of adiponectin. Nat Med. 2011;17(1):55-63.

22. Chaurasia B, Summers SA. Ceramides — lipotoxic inducers of metabolic disorders. Trends Endocrinol Metab. 2015;26(10):538-550.

23. Aerts JM, et al. Pharmacological inhibition of glucosylceramide synthase enhances insulin sensitivity. Diabetes. 2007;56(5):1341-1349

24. Zhao H, et al. Inhibiting glycosphingolipid synthesis improves glycemic control and insulin sensitivity in animal models of type 2 diabetes. Diabetes. 2007;56(5):1210-1218. 
25. Wentworth JM, et al. GM3 ganglioside and phosphatidylethanolamine-containing lipids are adipose tissue markers of insulin resistance in obese women. Int J Obes (Lond). 2016;40(4):706-713.

26. Bergman BC, et al. Muscle sphingolipids during rest and exercise: a C18:0 signature for insulin resistance in humans. Diabetologia. 2016;59(4):785-798.

27. Chavez JA, Siddique MM, Wang ST, Ching J, Shayman JA, Summers SA. Ceramides and glucosylceramides are independent antagonists of insulin signaling. J Biol Chem. 2014;289(2):723-734.

28. Bozic J, et al. Ganglioside GM3 content in skeletal muscles is increased in type 2 but decreased in type 1 diabetes rat models: implications of glycosphingolipid metabolism in pathophysiology of diabetes. J Diabetes. 2018;10(2):130-139.

29. Hornemann T, et al. The SPTLC3 subunit of serine palmitoyltransferase generates short chain sphingoid bases. J Biol Chem. 2009;284(39):26322-26330.

30. Han G, et al. Identification of small subunits of mammalian serine palmitoyltransferase that confer distinct acyl-CoA substrate specificities. Proc Natl Acad Sci U S A. 2009;106(20):8186-8191.

31. Russo SB, Tidhar R, Futerman AH, Cowart LA. Myristate-derived d16:0 sphingolipids constitute a cardiac sphingolipid pool with distinct synthetic routes and functional properties. J Biol Chem. 2013;288(19):13397-13409.

32. Quehenberger O, et al. Lipidomics reveals a remarkable diversity of lipids in human plasma. J Lipid Res. 2010;51(11):3299-3305.

33. Thusitha UA. Occurrence, structure elucidation, biosynthesis, functions and synthesis of sphingadienes. Mini-Rev Org Chem. $2015 ; 12(3): 282-292$

34. Véret J, et al. Ceramide synthase 4 and de novo production of ceramides with specific $\mathrm{N}$-acyl chain lengths are involved in glucolipotoxicity-induced apoptosis of INS-1 $\beta$-cells. Biochem J. 2011;438(1):177-189.

35. Turner N, et al. A selective inhibitor of ceramide synthase 1 reveals a novel role in fat metabolism. Nat Commun. 2018;9(1):3165

36. Huynh K, et al. High-Throughput Plasma Lipidomics: Detailed Mapping of the Associations with Cardiometabolic Risk Factors. Cell Chem Biol. 2019;26(1):71-84.e4.

37. Lemaitre RN, et al. Circulating sphingolipids, insulin, HOMA-IR, and HOMA-B: The Strong Heart Family Study. Diabetes. 2018;67(8):1663-1672.

38. Burla B, et al. MS-based lipidomics of human blood plasma: a community-initiated position paper to develop accepted guidelines. J Lipid Res. 2018;59(10):2001-2017.

39. Nelson JC, Jiang XC, Tabas I, Tall A, Shea S. Plasma sphingomyelin and subclinical atherosclerosis: findings from the multi-ethnic study of atherosclerosis. Am J Epidemiol. 2006;163(10):903-912.

40. Begum $\mathrm{H}$, et al. Discovering and validating between-subject variations in plasma lipids in healthy subjects. Sci Rep 2016;6:19139.

41. Hicks AA, et al. Genetic determinants of circulating sphingolipid concentrations in European populations. PLoS Genet. 2009;5(10):e1000672.

42. Tan KHX, et al. Cohort Profile: The Singapore Multi-Ethnic Cohort (MEC) study. Int J Epidemiol. 2018;47(3):699-699j.

43. Kathiresan S, et al. Six new loci associated with blood low-density lipoprotein cholesterol, high-density lipoprotein cholesterol or triglycerides in humans. Nat Genet. 2008;40(2):189-197.

44. Saw WY, et al. Establishing multiple omics baselines for three Southeast Asian populations in the Singapore Integrative Omics Study. Nat Commun. 2017;8(1):653.

45. Burla B, Muralidharan S, Wenk MR, Torta F. Sphingolipid analysis in clinical research. Methods Mol Biol. 2018;1730:135-162. 